\title{
Novel Experimental Agents for the Treatment of Hypercholesterolemia
}

This article was published in the following Dove Press journal:

Journal of Experimental Pharmacology

\author{
Ivan Pećin ${ }^{1,2}$ \\ Željko Reiner ${ }^{1,2}$
}

'Zagreb School of Medicine, University of Zagreb, Zagreb, Croatia; ${ }^{2}$ Division of Metabolic Diseases, Department of Internal Medicine, University Hospital Center, Zagreb, Croatia
Correspondence: Željko Reiner Division of Metabolic Diseases, Department of Internal Medicine, University Hospital Center Zagreb Kispaticeva 12, Zagreb, 10 000, Croatia Tel +385 | 2388772

Fax + 385 I 2388623

Email zreiner@kbc-zagreb.hr
Abstract: Atherosclerotic cardiovascular diseases (ASCVD) are still the leading cause of morbidity and mortality in most developed countries and even more in developing countries. Dyslipidemia is a well known main risk factor for ASCVD. Lipid-lowering treatment, particularly lowering LDL-cholesterol (LDL-C), can decrease the risk for ASCVD. New data and guidelines based upon them suggest that we should go with LDL-C levels as low as we can. Therefore, conventional lipid lowering agents (statins and statins+ezetimibe) are not enough mainly because of poor compliance and statin intolerance which is in the real world mostly pseudo-intolerance. PCSK9 inhibitors provided a new hope to further decrease LDL$\mathrm{C}$ but are still expensive, they have to be injected subcutaneously twice a month and their long-lasting adverse effects are not known. Therefore, there is a constant need to develop novel, more potent, more safe, less expensive, more user friendly regimens of hypolipemic agents (bempedoic acid, selective PPAR alpha receptor modulators etc). One of the ways to overcome poor compliance and increase the potency of therapy with less adverse effects are fixed combinations of established drugs (statin+ezetimibe). The future of hypolipemic agents is based on antisense therapy, ie. the use of specific oligonucleotide sequences blocking the translation of the selected protein (targeting apolipoprotein CIII, lipoprotein (a), apolipoprotein B) or RNA silencing technique (PCSK9 mRNA) and are in various stages of clinical trials. Some of them are almost ready to use in everyday clinical practice. High risk and very high risk patients (eg. familial hypercholesterolemia, familial severe chylomicronemia syndrome) will benefit most. The aim of this review is to inform about novel hypolipemic agents - potent and safe drugs for dyslipidemia which should reduce the risk of ASCVD.

Keywords: dyslipidemia, novel hypolipemic drugs, cardiovascular risk, LDL-cholesterol, triglycerides, inclisiran, PCSK9 inhibitors, pemafibrate, bempedoic acid, ANGPTL3, pelacarsen

\section{Introduction}

Atherosclerotic cardiovascular diseases (ASCVD), mainly caused by poor lifestyle, uncontrolled dyslipidemia, diabetes, smoking, and arterial hypertension, remain one of the leading causes of morbidity and mortality in most of the developed countries and even more in the developing countries. ${ }^{1}$ Low-density lipoprotein cholesterol (LDL-C) is a well established risk factor for development of ASCVD and for decades hypolipemic therapy was tailored to lower it. ${ }^{2}$ After the discovery that inhibition of 3-hydroxy-3-methylglutaryl-coenzyme A reductase (HMG-CoA) interferes with cholesterol synthesis in hepatocytes, thus decreasing LDL-C in the plasma, statins, as the first potent hypolipemic drugs, were developed. ${ }^{3}$ Their beneficial (not only lipid lowering but pleiotropic as well) ${ }^{4}$ effects have been 
shown in a number of studies and meta-analyses. One of the landmark studies was the Cholesterol Treatment Trialists (CTT) study which pointed that for every 1 $\mathrm{mmol} / \mathrm{L}$ LDL-C reduction there is a proportional reduction of major vascular events (myocardial infarction, coronary death, non-fatal myocardial infarction, stroke (MACE)) of about $20 \%{ }^{5}$ On the other hand there was always a question of whether LDL-C lowering to very low concentrations might have potential adverse effects since cholesterol is a necessary substance for cell membranes, steroid hormones, and bile salts. ${ }^{6}$ Nowadays it is quite clear that even very low levels of LDL-C do not cause any harm and none of the major safety concerns (cancer and depression) were confirmed except for an increased risk of new onset diabetes mellitus in patients with prediabetes after long-lasting treatment, but the effect of LDL-C lowering by far exceeds this adverse effect. Moreover, a lipid lowering concept based on inhibition of serin protease proprotein convertase subtilisin/kexin type 9 (PCSK9) with monoclonal antibodies alirocumab and evolocumab showed that very low LDL-C levels could be achieved (even $0.5 \mathrm{mmol} / \mathrm{L}$ and lower) with an adverse effect comparable to placebo ${ }^{7-10}$ but with great clinical benefit and reduction of major cardiovascular adverse events (MACE) and cardiovascular mortality.

Statins inhibit cholesterol synthesis in hepatocytes which causes two major compensating mechanisms. The first one is upregulation of a low-density lipoprotein receptor (LDL-R) on the hepatic cells surface which increases LDL-C uptake and decreases serum LDL-C levels, and the second is an increase in cholesterol absorption in the intestine. This later compensating mechanism is not favorable and therefore drugs which inhibit cholesterol absorption were developed. Ezetimibe is a drug that acts primarily by blocking dietary cholesterol absorption in the small intestine. It inhibits cholesterol transport protein in enterocytes called Niemann-Pick C1-like1 (NPC1L1). This action results in more potent LDL-R expression and recirculation with greater LDL-C reduction when combined with statins than statins alone can achieve. ${ }^{11}$ A landmark study that confirmed the beneficial effect of ezetimibe combined with statin (simvastatin) was the IMPROVE-IT trial (The Improved Reduction of Outcomes: Vytorin Efficacy International Trail). This trial showed that ezetimibe, when added to statin therapy, reduces more LDL-C than statin alone and improves cardiovascular outcomes. These results positioned addition of ezetimibe to statin treatment as "conditio sine qua non" in therapy of high-risk patients in secondary prevention and in patients that need LDL-C reduction up to $20 \%$ and do not tolerate statin. ${ }^{12}$ In real life statins, despite proven clear clinical benefits of their use, are mostly prescribed in too low doses and there is a tendency to use less potent statins like simvastatin and fluvastatin, despite evidence that the more potent statins rosuvastatin and atorvastatin are much more appropriate choices. ${ }^{13}$ Also, ezetimibe is added to statin treatment much less frequently than it should be, particularly in high risk and very high risk patients.

A factor that is often used as an explanation (or better to say, excuse) for using too low doses of statins is statin associated myopathy and intolerance to statins in general. ${ }^{14,15}$ However, in real life this is more often pseudointolerance to statins, but it also has an impact on statin prescription and compliance to treatment. ${ }^{16}$ Wood et al ${ }^{17}$ have checked whether adverse effects would be induced by a statin or placebo. A total of 60 patients were randomized. They have concluded that in patients who had discontinued statin therapy because of adverse effects, $90 \%$ of the symptom burden elicited by a statin challenge was also elicited by placebo. Half of the study patients were able to successfully restart statin therapy.

Monoclonal antibodies alirocumab and evolocumab, inhibitors of PCSK9, further confirmed and underlined the concept of safe lowering of LDL-C to very low levels, achieving significant clinical benefit - reduction of MACE and cardiovascular mortality. Nowadays we cannot call them "novel therapies" because they have been in clinical use for the last 3-4 years but unfortunately they are still not available in all countries or are not reimbursed and therefore are used much less than one would expect. ${ }^{18,19}$ PCSK9 inhibitors brought additional hope and showed a significant benefit for the patients with severe dyslipidemias, particularly those with familial hypercholesterolemia (FH) (both homozygous and heterozygous). With evolocumab and alirocumab above maximally tolerated statin treatment with and/or without ezetimibe a vast majority of FH patients were finally able to achieve target LDL-C values in primary and secondary prevention. ${ }^{20,21}$ There is clear evidence that lipid modifying therapy is more and more potent with an excellent safety profile and achieving favorable clinical outcomes.

Before we proceed with novel therapies that are on the horizon we need to underline once again lifestyle measures as a first tool in the treatment of dyslipidemias. ${ }^{22}$ For example, there is a clear benefit of physical activity. 
Moreover, physical activity/exercise when compared with the control group produced increased telomerase activity and telomerase length which is important for healthy aging. Smoking is also a very well known risk factor. Its negative consequences were proven in many studies, from the Framingham study to Seven countries study and in a lot of more recent studies dealing with modifiable risk factors. $^{23,24}$ Therefore it is difficult to understand why a significant percentage of patients who have survived a MACE do not give up smoking and do not have any intention to give up smoking. ${ }^{25,26}$ This main aim of this review is to present novel potential agents that beside LDL-C lowering and ASCVD risk reduction might have influence on other lipid parameters such as elevated triglycerides or lipoprotein (a) [Lp (a)] which also have an impact on ASCVD risk, particularly when they are combined with other risk factors.

\section{Novel but Almost Established Therapies}

Because of the big gap between available therapy, therapy use, compliance, and cardiovascular outcomes in real life, ${ }^{27}$ there is a persistent need to develop new drugs that will overcome barriers and obstacles which interfere with current problems. These drugs should be more effective than placebo (active therapy), they should be safe, easier to use by medical personnel and patients, and finally not too expensive. There are several hypolipemic drugs that are in the stage II and III trials. LDL-C, triglycerides, and Lp (a) are the main targets for these drugs. Old and novel agents and their main characteristics are presented in Table 1.

\section{Inclisiran}

Since 2003 when it was noticed that gene variation on PCSK9 (gain of function) resulted in inherited high cholesterol levels, clinically manifested as $\mathrm{FH}$, the concept of PCSK9 inhibition came into focus as a new and promising concept of lipid lowering. ${ }^{28}$

The concept of PCSK9 inhibition showed beneficial results (in FOURIER and ODYSSEY OUTCOMES studies) and therefore other strategies of PCSK9 inhibition were examined as well. Intracellular inhibition of PCSK9 by a small interfering RNA (siRNA) is currently in the focus of clinical research. This way of PCSK9 inhibition is specially tailored to hepatic intracellular PCSK9 inhibition. Specific N-acetylgalactosamine modification of the double stranded inclisiran molecule ensures hepatic uptake via asialoglycoprotein receptors expressed only on hepatic cells. After uptake, inclisiran is bounded to RNA-induced silencing complex in hepatocyte cytoplasm interfering with the production of PCSK9. ${ }^{29}$ Inclisiran is along acting siRNA targeting PCSK9 that is administered by subcutaneous injections in quite favorable schemes (0-90-180 days) which has an advantage when compared with PSCK9 inhibition produced by monoclonal antibodies (subcutaneous injections every 14 days or in some cases as an option one injection per month). Efficacy and safety of siRNA concept was proved by ORION trials. However, the results of an ongoing outcome trail are still awaited. ${ }^{30}$ ORION 10 and -11 trials focused on patients with ASCVD. More than 3,000 patients were enrolled (1,561 in ORION 10 and 1,617 in ORION 11) with mean LDL-C levels at baseline $2.71 \pm 0.99 \mathrm{mmol} / \mathrm{L}(104.7 \pm 38.3 \mathrm{mg} / \mathrm{dL})$ and $2.73 \pm 1.01 \mathrm{mmol} / \mathrm{L}(105.5 \pm 39.1 \mathrm{mg} / \mathrm{dL})$, respectively. Inclisiran reduced LDL-C levels by $49.2-53.8 \%$ compared to placebo, respectively. Adverse events were generally similar in the inclisiran and placebo groups in both trials. The only difference were injection-site reactions that were generally mild, and none were persistent when compared with the placebo group $(2.6 \%$ vs $0.9 \%$ in the ORION-10 trial and $4.7 \%$ vs $0.5 \%$ in the ORION-11 trial). The benefit of this treatment when compared with PCSK9 inhibition by monoclonal antibodies is that inclisiran is administered subcutaneously every 6 months but, as already mentioned, hard outcome data are still awaited. Inclisiran has been submitted for FDA and EMA approval in adults with ASCVD or heterozygous $\mathrm{FH}$ patients who have elevated LDL-C.

\section{Bempedoic Acid}

Bempedoic acid is a prodrug that has a lipid lowering effect by inhibition of adenosine triphosphate citrate lyase in the liver. ${ }^{31}$ The research of bempedoic acid was driven by the fact that a significant number of patients do not tolerate statins (although in most of the cases it is pseudo-intolerance). Nevertheless, there is a constant urge to lower LDL-C in ways beyond traditional hypolipemic drugs like statins, maybe to avoid the adverse effects of statins.

Bempedoic acid's chemical name is 8-hydroxy2,2,14,14-tetramethylpentadecanedioic acid. Adenosine triphosphate-citrate lyase (ACL) is an enzyme upstream of 3-hydroxy-3-methyl-glutaryl-coenzyme A reductase. Inhibiting ACL bempedoic acid creates less substrate for cholesterol and fatty acid synthesis, thus decreasing liver 
Table I Summary of the Lipid Lowering Agents Mentioned in the Paper

\begin{tabular}{|c|c|c|c|c|c|}
\hline Agent & $\begin{array}{l}\text { Mechanism of } \\
\text { Action }\end{array}$ & $\begin{array}{l}\text { Main Lipid } \\
\text { Lowering } \\
\text { Effect }\end{array}$ & $\begin{array}{l}\text { Administration } \\
\text { Scheme }\end{array}$ & Side-Effects & Comment \\
\hline Statin & HMG-CoA inhibition & LDL - C & Ix/day p.o. & $\begin{array}{l}\text { Myopathy, } \\
\text { increased liver } \\
\text { enzymes }\end{array}$ & $\begin{array}{l}\text { Side-effects are rare, novel statins like } \\
\text { rosuvastatin and atorvastatin can be taken in } \\
\text { the morning because of long } \mathrm{t} I / 2\end{array}$ \\
\hline Ezetimibe & $\begin{array}{l}\text { NPCILI protein } \\
\text { inhibition }\end{array}$ & LDL-C & Ix/day p.o. & Diarrhoea & Side-effects are rare \\
\hline $\begin{array}{l}\text { PCSK9i } \\
\text { (alirocumab/ } \\
\text { evolocumab) }\end{array}$ & PCSK9 inhibition & LDL-C & $\begin{array}{l}2 x / \text { month }(|x| \\
\text { month) s.c. }\end{array}$ & $\begin{array}{l}\text { Injection site } \\
\text { reactions }\end{array}$ & Side-effects are rare, not more than placebo \\
\hline Inclisiran & $\begin{array}{l}\text { siRNA targeting } \\
\text { mRNA PCSK9 }\end{array}$ & LDL-C & $2 x / y e a r$ s.c. & $\begin{array}{l}\text { Injection site } \\
\text { reactions }\end{array}$ & $\begin{array}{l}\text { Side-effects are rare, not more than placebo } \\
\text { (still under investigation) }\end{array}$ \\
\hline $\begin{array}{l}\text { Bempedoic } \\
\text { acid }\end{array}$ & $\begin{array}{l}\text { Inhibiting } A C L \text { and } \\
\text { AMPK }\end{array}$ & LDL-C & I/day p.o. & $\begin{array}{l}\text { Not greater than } \\
\text { placebo }\end{array}$ & Alernative to SAMS? \\
\hline $\begin{array}{l}\text { Icosapent } \\
\text { ethyl }\end{array}$ & LPL? & TGs & I/day p.o. & $?$ & $\begin{array}{l}\text { Benefit of long-term use of this agent still } \\
\text { needs to be proven; many pleiotropic effects }\end{array}$ \\
\hline Volanesorsen & $\begin{array}{l}\text { Antisense } \\
\text { oligonucleotide to } \\
\text { apo C-III }\end{array}$ & TGs & $2 x /$ year s.c. & $\begin{array}{l}\text { Thrombocytopenia } \\
\text { and injection-site } \\
\text { reactions }\end{array}$ & Treatment of ultra rare LPL deficiency \\
\hline ANGPTL3 & $\begin{array}{l}\text { Monoclonal anti- } \\
\text { ANGPLT3 antibody } \\
\text { and ASO }\end{array}$ & TGs, LDL-C & $2 x / y e a r$ s.c.? & $\begin{array}{l}\text { Not yet fully } \\
\text { determined }\end{array}$ & Studies are ongoing \\
\hline Pemafibrate & $\begin{array}{l}\text { Peroxisome } \\
\text { proliferator-activated } \\
\text { receptor alpha } \\
\text { modulator }\end{array}$ & TGs & I/day p.o. & Liver enzymes? & $\begin{array}{l}\text { Clinical data as well as long-term efficacy } \\
\text { and safety need to be investigated }\end{array}$ \\
\hline Pelacarsen & $\begin{array}{l}\text { ASO to } \\
\text { apolipoprotein(a) }\end{array}$ & $\operatorname{Lp}(\mathrm{a})$ & $2 x / y e a r$ s.c.? & $?$ & The agent is in phase III trial \\
\hline
\end{tabular}

Abbreviations: HMG-CoA, 3-hydroxy-3-methylglutaryl-coenzyme A reductase; LDL-C, Low density lipoprotein cholesterol; NPCILI, Niemann-pick-CI like-I protein; PCSK9i, inhibitor of proprotein kexin serin convertase type 9; p.o., peroral therapy; s.c., subcutaneous therapy; ACL, Adenosine triphosphate-citrate lyase; AMPK, adenosine monophosphate-activated protein kinase; SAMS, statin associated muscular symptoms; TGs, Triglycerides; LPL, lipoprotein-lipase; Apo-CIII, Apolipoprotein CIII; ANGPTL3, Angiopoietin-Like 3; ASO, anti sense oligonucleotide; Lp(a), lipoprotein (a).

Notes: ? indicates unknown side effects.

cholesterol synthesis and serum LDL-C levels via upregulating LDL-R. ${ }^{32}$ Bempedoic acid also has effects on adenosine monophosphate-activated protein kinase (AMPK). In a manner complementary to the effects of ACL, the activation of AMPK results in inhibitory phosphorylation of acetylCoA carboxylase and HMG-CoA reductase. Therefore, bempedoic acid affects multiple phases of the lipid-synthesis pathway, it inhibits sterol and fatty acid synthesis, and increases mitochondrial long-chain fatty acid oxidation. ${ }^{33}$

The effect of bempedoic acid can only be achieved if activated by acyl-CoA synthetase I (ACSVL1) to ETC-1002-
CoA and ESP15228-CoA. A very important fact is that ACSVL1 is present only in the liver but not in skeletal muscles. This decreases the risk for muscle-related adverse effects. Based on this potential advantage to statin therapy, bempedoic acid was tested in several trials on animals and humans as well as in clinical trials. The study on healthy volunteers demonstrated a mean decrease in LDL-C levels of $36 \%$ when compared with placebo. ${ }^{34}$ Many Phase II trials with bempedoic acid monotherapy and combined therapy regimes were followed by robust Phase III trials. Thompson et $\mathrm{al}^{35}$ reported that hs-CRP levels significantly 
decreased by $42 \%$ in patients treated with bempedoic acid. The same authors treated patients with dyslipidemia who were statin intolerant with bempedoic acid and concluded that bempedoic acid monotherapy or in combination with ezetimibe is effective and safe in patients with statin intolerance (but also in patients without statin intolerance) ${ }^{36}$ reported that hs-CRP levels significantly decreased by $42 \%$ in patients treated with bempedoic acid. The same authors treated patients with dyslipidemia who were statin intolerant with bempedoic acid and concluded that bempedoic acid monotherapy or in combination with ezetimibe is effective and safe in patients with statin intolerance (but also in patients without statin intolerance). The same authors adverse events, particularly those muscle-related, and discontinuations of treatment because of adverse effects in patients treated with bempedoic acid and statins were similar to those treated with statins and placebo. ${ }^{37}$

After promising results of CLEAR HARMONY, WISDOM, SERENITY, and TRANQUILITY studies (phase III, efficacy and safety of bempedoic acid alone or in combination with ezetimibe and/or statin in patients with ASCVD, heterozygous familial hypercholesterolemia (HeFH)), FDA approved on February 21, 2020 bempedoic acid in addition to statin therapy for adult patients with $\mathrm{HeFH}$ or established HeFH and EMA approved it on April 6, 2020 for the same types of patients. ${ }^{38}$ The CLEAR OUTCOME study is a multicenter randomized double-blind, placebo controlled study with the main aim to provide an answer to the question of whether statin intolerant patients with ASCVD can benefit in terms of clinical outcomes when treated with bempedoic acid. The study is ongoing and has enrolled 14,014 high risk patients. The final results are expected to be published about the same time as this article. ${ }^{39}$ An advantage or disadvantage, depending upon the point of view, of bempedoic acid is that it is taken orally.

\section{Icosapent Ethyl}

While the debate whether lowering of serum triglycerides (TGs) does produce any benefits is still ongoing, ${ }^{40,41}$ new drugs are being developed that act on TGs. Namely, the results of some authors clearly showed that in patients with residual risk for ASCVD with elevated TGs levels, TGs are at least an independent marker for an increased risk of ischemic events as shown by Mendelian randomization studies. ${ }^{42}$ One of these substances is icosapent ethyl, a highly purified eicosapentaenoic acid ethyl ester, that lowers TGs levels, but the benefit of long-term use of this substance still needs to be proven. Bhatt et $\mathrm{al}^{43}$ performed a multicenter, randomized, double-blind, placebocontrolled trial involving patients with established ASCVD or with diabetes and other risk factors. The trial was named The Reduction of Cardiovascular Events with Icosapent Ethyl-Intervention Trial (REDUCE-IT). Overall 8,179 patients were enrolled, as already mentioned these were mostly secondary prevention patients, and were followed for a median of 4.9 years. They were also receiving statin therapy. The patients were randomly assigned to receive $2 \mathrm{~g}$ of icosapent ethyl twice daily (total daily dose $4 \mathrm{~g}$ ) or placebo. Their fasting TGs levels were 135-499 mg/ $\mathrm{dL}(1.52-5.63 \mathrm{mmol} / \mathrm{L})$ and LDL-C levels were $41-100 \mathrm{mg} /$ dL (1.06-2.59 mmol/L). A primary end-point event occurred in $17.2 \%$ of the patients from the icosapent ethyl group, as compared with $22.0 \%$ of the patients in the placebo group which was a significant difference. Nevertheless, a larger percentage of patients in the icosapent ethyl group than in the placebo group were hospitalized for atrial fibrillation or flutter $(3.1 \%$ vs $2.1 \%, P=0.004)$. Serious bleeding events did not differ significantly between those two groups. The conclusion was that in patients with elevated TGs levels despite the use of statins, the risk of ischaemic events, including cardiovascular death, was significantly lower among those who received $2 \mathrm{~g}$ of icosapent ethyl twice daily than among those who received placebo. These significant cardiovascular mortality benefits of purified EPA ethyl ester, with a $25 \%$ relative risk reduction in major cardiovascular events were the reason why it was initially approved by FDA in 2012 for adults with severe hypertriglyceridemia, but on December 13, 2019 it was approved as an adjunctive therapy to reduce the risk of cardiovascular events among adults with elevated TGs levels and either established ASCVD or diabetes and two or more additional risk factors for ASCVD.

\section{Volanesorsen}

Volanesorsen is an antisense oligonucleotide to apolipoprotein C-III (Apo-CIII). ${ }^{44}$ Apo-CIII is a multifunctional protein which participates in the metabolism of TGs, and is responsible for various pleiotropic actions like endothelial cell dysfunction and inflammation, dysfunction of pancreatic beta cells, and counteraction of potential antiatherogenic effect of HDL particle components. The levels of TGs can be lowered using antisense oligonucleotides directed against Apo-CIII messenger RNA and this approach resulted in developing a new drug - volanesorsen. Its efficacy in lowering TGs has been shown in 
various experimental models and later in clinical studies. Volanesorsen was granted a conditional marketing authorisation by EMA in May 2019 for ultra-rare familial chylomicronaemia syndrome (FCS). ${ }^{45}$ Witztum et $\mathrm{al}^{46}$ recently published data from a phase III trial including 66 patients with FCS who were treated and followed for 52 weeks. At 3 months, $77 \%$ of the patients in the volanesorsen group, as compared with $10 \%$ of patients in the placebo group, had TGs levels less than $8.5 \mathrm{mmol} / \mathrm{L}$. However, thrombocytopenia and injection-site reactions were common adverse events. Thrombocytopenia as a potentially serious adverse effect raised questions about volanesorsen safety. ${ }^{47}$ Nevertheless, volanesorsen has a specific role in the treatment of the ultra rare patients with FCS.

\section{Lipid Lowering Drugs Which are Not Yet on the Market Angiopoietin-Like 3 (ANGPTL3)}

Recent genetic studies revealed yet another gene and its product that have a significant role in the lipoprotein mechanism. Angiopoietin-like 3 gene loss-of-function is associated with decreased plasma levels of TGs, LDL-C, and elevated highdensity lipoprotein cholesterol (HDL-C). Therefore, it was proposed that blocking ANGPLT3 might produce a beneficial effect on cardiovascular risk and future outcomes. $\mathrm{Xu}$ et $\mathrm{al}^{48}$ performed RNAi-mediated gene silencing of ANGPTL3 in five mouse models and in human hepatoma cells. The authors validated results by deleting ANGPTL3 gene using the CRISPR/Cas9 genome editing system and have concluded that reduced secretion and increased uptake of ApoB-containing lipoproteins may contribute to the low LDL-C observed in mice and humans with genetic ANGPTL3 deficiency. Evinacumab, a monoclonal antiANGPLT3 antibody and anti-sense oligonucleotide (ASO), plays a pivotal role in ANGPLT3 inhibition. ANGPLT3 was discovered in 1999 by Conklin et $\mathrm{al}^{49}$ as a potent modulator of lipoprotein metabolism. Carriers of loss-of-function gene variation were associated with a $34 \%$ decrease in cardiovascular events, as was reported by Stitziel et al. ${ }^{50}$ The main action of ANGPLT3 after its secretion is that it targets the adipose tissue and muscles by activating lipolysis, increasing the release of glycerol and free fatty acids from adipocytes, and inhibiting the enzyme lipoprotein lipase (LPL), thus increasing TGs-rich lipoproteins. ${ }^{51}$ Aiming at this pathway several possibilities of ANGPLT3 inhibition were developed. The first concept with monoclonal antibody evinacumab showed a promising effect on lipid metabolism in mice. In vivo studies showed dosedependent reduction of TGs, total cholesterol (TC), LDL-C, and elevation of HDL-C after subcutaneous injection of evinacumab. The same effect was seen with an antisense oligonucleotide (ASO) ${ }^{52}$ Gaudet et $\mathrm{al}^{53}$ showed similar effects on high risk patients (patients with $\mathrm{HoFH}$ ), in which administration of evinacumab resulted in a nearly $50 \%$ reduction of LDLC, which was promising, especially for this type of patients. There are indications that ANGPLT3 antagonists lower LDL$\mathrm{C}$ by a mechanism which is independent of LDL-R, which might explain, at least partially, a strong effect in $\mathrm{HoFH}$ patients. As already mentioned above the interference with ANGPLT3 promises exciting effects on lipid parameters and for sure it will be in the focus of the research in the next years. Moreover, besides the effects of ANGPTL3 on lipid metabolism, the analysis of its specific protein structure has suggested possible additional effects such as an anti-inflammatory and anti-angiogenic, as well as an increase of macrophage cholesterol efflux which all could be a useful addition to its antiatherosclerotic effect.

\section{Pemafibrate}

Pemafibrate is a novel selective peroxisome proliferatoractivated receptor alpha modulator that has a beneficial role in patients with dyslipidemia. Its main effect is TGs lowering and according to recently published studies it shows better results than therapy with fenofibrate. ${ }^{54}$ It is well known that patients who have LDL-C levels are under control still have a substantially increased cardiovascular risk. Therefore, a constant need to deal with so called "residual risk" is basis of a conceptual framework and discussion about new therapeutic potentials elaborated in a consensus statement by IAS and R3i Foundation. ${ }^{55}$ Among other strategies this consensus paper underlines the potential benefits of a new drug pemafibrate, which is a PPAR $\alpha$ modulator (SPPARM $\alpha$ ) with extremely high selectivity to PPARs subtypes. The main potential advantage of this drug is to reduce the occurrence of adverse effects associated with fibrate-type drugs. Moreover, pemafibrate showed fewer restrictions for use in patients with renal disease than other fibrates (it is excreted by liver) and a better safety profile when combined with statins. Pemafibrate does not only have lipid-improving effects similar to those of existing fibrate-type drugs but it has pleiotropic effects such as anti-inflammatory effects, it has beneficial effects on animal models of NASH, and causes no increase in liver enzymes (ALT, AST) and serum creatinine. ${ }^{56}$ Ida et al, ${ }^{54}$ in a meta-analysis of seven randomized controlled trials (RCTs), showed that with respect to 
efficacy, TGs, HDL-C, and non-HDL-C levels as well as HOMA-IR improved in the pemafibrate group when compared with the placebo group. When PPAR $\alpha$ is activated in the liver, the expression of apolipoprotein C-III, which inhibits the activity of the TG-hydrolyzing lipoprotein lipase (LPL), decreases thereby causing increased LPL activity which causes decreased blood TGs levels. ${ }^{57}$ Furthermore, the activation of PPAR $\alpha$ induces the expression of apolipoprotein A-I and apolipoprotein A-II, which are the main apolipoproteins of HDL, and therefore the HDL-C level increases. It increases macrophage cholesterol efflux to HDL as well. It also improves HOMA index but it is still not clear to explain the improvement of insulin resistance as a response to pemafibrate.

The only unfavorable effect of pemafibrate is slight elevation of LDL-C. Therefore, although the increase of LDL-C levels following pemafibrate administration is expected to be safe, further studies are required to explain the clinical significance of this side-effect. Because of everything mentioned before, the results of a large-scale clinical trial [entitled "Pemafibrate to Reduce Cardiovascular Outcomes by Reducing Triglycerides in Patients with Diabetes (PROMINENT)"] aiming to assess the effect of pemafibrate on cardiovascular outcomes which is ongoing, are eagerly awaited. ${ }^{58}$ At the moment pemafibrate has marketing authorization only in Japan.

\section{Pelacarsen}

Pelacarsen or ISIS 681,257 , or IONIS APO (a)-LRx, or AKCEA-APO (a)-LRx or TQJ230 is hepatocyte-directed antisense oligonucleotide to apolipoprotein (a) [Apo (a)] developed to decrease $\mathrm{Lp}$ (a) targeting the $L P A$ gene messenger RNA, in patients with ASCVD. Lp (a) is a highly atherogenic, LDL-like lipoprotein containing Apo B linked by a disulphide bridge to Apo (a). ${ }^{59}$ Pelacarsen safely and dosedependently decreased Lp (a) levels by $35-80 \%$ and a Phase 3 trial [Lp(a) HORIZON] is ongoing starting from 2020 and is planned to end in $2024 .{ }^{60}$ In this trial 7,680 patients with established ASCVD are included and randomized to receive $80 \mathrm{mg}$ TQJ230 injected monthly or placebo. Major inclusion criteria are $\mathrm{Lp}$ (a) levels $>70 \mathrm{mg} / \mathrm{dL}$ and myocardial infarction or stroke ( $>3$ months from screening and randomization to $<10$ years prior to the screening visit) or clinically significant symptomatic PAD. The study has been designed to compare TQJ230 vs placebo in reducing the risk of expanded MACE (cardiovascular death, non-fatal MI, non-fatal stroke, and urgent coronary re-vascularization requiring hospitalization) while the second primary outcome measure is the same as above in patients with $\mathrm{Lp}(\mathrm{a}) \geq 90 \mathrm{mg} / \mathrm{dL}$. However, the question whether $\mathrm{Lp}$ (a) lowering against other LDL-C lowering therapy does really reduce the risk of ASCVD is still open. ${ }^{64}$

Stiekema et $\mathrm{al}^{61}$ reported that pro-inflammatory activation of circulating monocytes is a potential mechanism by which Lp(a) can also influence ASCVD. Data on 32 patients with ASCVD indicate that patients receiving Apo(a) antisense (AKCEA-APO(a)-LRx) (NCT03070782), due to elevated $L p(a)$ have a greater effect of $L p(a)$-lowering on gene expression and function of monocytes than the patients who received PCSK9 inhibitors. AKCEA-APO(a)-LRx lowered $\mathrm{Lp}$ (a) by $47 \%$ and reduced the pro-inflammatory gene expression in monocytes of patients with ASCVD who had elevated Lp(a), which coincided with a functional reduction in transendothelial migration capacity of monocytes ex vivo. This effect was superior than the effect of currently available PCSK9 inhibitors which did not alter neither transcriptome nor functional properties of monocytes but showed only a significant LDL-C reduction.

\section{Some New Promising Approaches}

It has been shown that clustered regularly interspaced short palindromic repeats (CRISPR)-Cas-mediated genome editing that relies on Cas9/sgRNA ribonucleoprotein complexes (RNPs) to target and edit DNA as well as CRISPR-Cas12a might be promising in dyslipidemia treatment. Most recently a new methodology based upon Cas9/sgRNA has been developed using modified lipid nanoparticles to efficiently deliver RNPs into cells and edit tissues. The developed carriers were also able to deliver RNPs to restore dystrophin expression in DMD mice and significantly decrease serum PCSK9 level. ${ }^{62}$ When PCSK9 was selected as the target gene based upon CRISPR-Cas12a genome editing, an efficient PCSK9 disruption occurred in vivo $(\sim 48 \%)$, significantly reducing the expression of PCSK9 and a $45 \%$ reduction of serum cholesterol. ${ }^{63}$ Chadwick et al ${ }^{65}$ used the third generation base editor (BE3) delivered via adenoviral vectors and have produced a generation of ANGPTL3 loss-of-function mutations in mice. Serum TGs and total cholesterol levels were significantly reduced in the BE3-ANGPTL3 group when compared with the controls.

\section{Conclusion}

Atherosclerosis is the disease which causes clinical ASCVD. ASCVD is caused by the multifactorial interaction of various conditions (modifiable but also non-modifiable). Dyslipidemia is widely recognized as one of the most important risk factors in this process. In most cases dyslipidemia can be effectively 
treated if an adequate dose of hypolipemic drugs are used and if adherence and compliance are adequate. Statins, which have been available for almost four decades, decrease LDL-C and ASCVD morbidity as well as mortality and are therefore pivotal in ASCVD prevention, both primary and secondary. Ezetimibe is a complementary drug to statins acting by inhibiting cholesterol intestinal absorption and should be used in combination with a statin. Fixed combination of both drugs in adequate doses will achieve better adherence and a better lipid lowering effect. However, ASCVD still remains the leading cause of mortality worldwide, which suggests that additional lipid-lowering therapies are needed. This review discusses only agents that have ether just appeared on some markets or are "ante portas" and that will for sure improve and revolutionize anti-atherosclerotic drug therapy. There are many other substances in the pipelines of drug industries which might prove in the future to be useful, but for the sake of space we have not discussed those which are in Phase I or II.

\section{Disclosure}

IP reports speaker honoraria from Sanofi Genzyme, Takeda, Amgen and advisory board for Novartis, outside the submitted work. ZR reports speaker honoraria from Akcea, outside the submitted work. The authors report no other potential conflicts of interest in this work.

\section{References}

1. Mach F, Baigent C, Catapano AL, et al. ESC Scientific Document Group, 2019 ESC/EAS Guidelines for the management of dyslipidaemias: lipid modification to reduce cardiovascular risk: the Task Force for the management of dyslipidaemias of the European Society of Cardiology (ESC) and European Atherosclerosis Society (EAS). Eur Heart J. 2020;41:111, 188. doi:10.1093/eurheartj/ehz455

2. Castelli WP. Epidemiology of coronary heart disease: the Framingham study. Am J Med. 1984;76(2A):4-12. doi:10.1016/0002-9343(84) 90952-5

3. Reiner Ž. Statins in the primary prevention of cardiovascular disease. Nat Rev Cardiol. 2013;10(8):453-464. doi:10.1038/nrcardio.2013.80

4. Sahebkar A, Pećin I, Tedeschi-Reiner E, et al. Effects of statin therapy on augmentation index as a measure of arterial stiffness: A systematic review and meta-analysis. Int $J$ Cardiol. 2016;212:160-168. doi:10.1016/j.ijcard.2016.03.010

5. Baigent C, Blackwell L, Emberson J, et al.; Cholesterol Treatment Trialists' (CTT) Collaboration. Efficacy and safety of more intensive lowering of LDL cholesterol: a meta-analysis of data from 170,000 participants in 26 randomised trials. Lancet. 2010;376 (9753):1670-1681. doi:10.1016/S0140-6736(10)61350-5.).

6. Ben-Yehuda O, DeMaria AN, Low LDL-C. levels and cancer reassuring but still not definitive. $J$ Am Coll Cardiol. 2008;52(14):1150-1151. doi:10.1016/j.jacc.2008.08.001

7. Sabatine MS, Giugliano RP, Keech AC, et al.; FOURIER Steering Committee and Investigators. Evolocumab and clinical outcomes in patients with cardiovascular disease. $N$ Engl J Med. 2017;376 (18):1713-1722. doi:10.1056/NEJMoa1615664.
8. Schwartz GG, Steg PG, Szarek M, et al.; ODYSSEY OUTCOMES Committees and Investigators. Alirocumab and cardiovascular outcomes after acute coronary syndrome. $N$ Engl J Med. 2018;379 (22):2097-2107. doi:10.1056/NEJMoa1801174.

9. Jukema JW, Zijlstra LE, Bhatt DL, et al.; ODYSSEY OUTCOMES Investigators. Effect of alirocumab on stroke in odyssey outcomes. Circulation. 2019;140(25):2054-2062. doi:10.1161/CIRCULATIO NAHA.119.043826.

10. Giugliano RP, Pedersen TR, Park JG, et al.; FOURIER Investigators. Clinical efficacy and safety of achieving very low LDL-cholesterol concentrations with the PCSK9 inhibitor evolocumab: a prespecified secondary analysis of the FOURIER trial. Lancet. 2017;390 (10106):1962-1971. doi: 10.1016/S0140-6736(17)32290-0.

11. Phan BA, Dayspring TD, Toth PP. Ezetimibe therapy: mechanism of action and clinical update. Vasc Health Risk Manag. 2012;8:415-427. doi:10.2147/VHRM.S33664

12. Cannon CP, Blazing MA, Giugliano RP, et al.; IMPROVE-IT Investigators. Ezetimibe added to statin therapy after acute coronary syndromes. $N$ Engl J Med. 2015;372(25):2387-2397. doi:10.1056/ NEJMoa1410489.

13. Pedersen TR, Faergeman O, Kastelein JJ, et al.; Incremental Decrease in End Points Through Aggressive Lipid Lowering (IDEAL) Study Group. High-dose atorvastatin vs usual-dose simvastatin for secondary prevention after myocardial infarction: the IDEAL study: a randomized controlled trial. JAMA. 2005;294(19):2437-2445. doi:10.1001/jama.294.19.2437.

14. Reiner Ž. Resistance and intolerance to statins. Nutr Metab Cardiovasc Dis. 2014;24(10):1057-1066. doi:10.1016/j.numec d.2014.05.009

15. Šimić I, Reiner Ž. Adverse effects of statins - myths and reality. Curr Pharm Des. 2015;21(9):1220-1226. doi:10.2174/138161282066614 1013134447

16. Banach M, Rizzo M, Toth PP, et al. Statin intolerance - an attempt at a unified definition. Position paper from an international lipid expert panel. Arch Med Sci. 2015;11(1):1-23. doi:10.5114/aoms.2015.49807

17. Wood FA, Howard JP, Finegold JA, et al. N-of-1 trial of a statin, placebo, or no treatment to assess side effects. $N$ Engl $J$ Med. 2020;383(22):2182-2184. doi:10.1056/NEJMc2031173

18. Reiner Ž. PCSK9 inhibitors - past, present and future. Expert Opin Drug Metab Toxicol. 2015;11(10):1517-1521. doi:10.1517/ 17425255.2015.1075506.

19. Reiner Ž. PCSK9 inhibitors in clinical practice: expectations and reality. Atherosclerosis. 2018;270:187-188. doi:10.1016/j. atherosclerosis.2018.01.001

20. Kastelein JJ, Ginsberg HN, Langslet G, et al. ODYSSEY FH I and FH II: 78 week results with alirocumab treatment in 735 patients with heterozygous familial hypercholesterolaemia. Eur Heart J. 2015;36 (43):2996-3003. doi:10.1093/eurheartj/ehv370

21. Raal FJ, Honarpour N, Blom DJ, et al.; TESLA Investigators. Inhibition of PCSK9 with evolocumab in homozygous familial hypercholesterolaemia (TESLA Part B): a randomised, double-blind, placebo-controlled trial. Lancet. 2015;385 (9965):341-350. doi:10.1016/S0140-6736(14)61374-X.).

22. Reiner $\breve{Z}$, Laufs U, Cosentino F, et al. The year in cardiology 2018: prevention. Eur Heart J. 2019;40(4):336-344. doi:10.1093/eurheartj/ ehy894

23. Tindle HA, Stevenson Duncan M, Greevy RA, et al. Lifetime smoking history and risk of lung cancer: results from the framingham heart study. J Natl Cancer Inst. 2018;110(11):1201-1207. doi:10.1093/jnci/ djy041

24. Keys A, Menotti A, Karvonen MJ, et al. The diet and 15-year death rate in the seven countries study. Am J Epidemiol. 1986;124 (6):903-915. doi:10.1093/oxfordjournals.aje.a114480.)

25. Reiner Ž. The importance of smoking cessation in patients with coronary heart disease. Int J Cardiol. 2018;258:26-27. doi:10.1016/ j.ijcard.2018.02.009 
26. Prugger C, Wellmann J, Heidrich J, et al.; EUROASPIRE Study Group. Readiness for smoking cessation in coronary heart disease patients across Europe: results from the EUROASPIRE III survey. Eur J Prev Cardiol. 2015;22(9):1212-1219. doi:10.1177/ 2047487314564728.).

27. De Backer G, Jankowski P, Kotseva K; EUROASPIRE $\mathrm{V}$ collaborators, et al. Management of dyslipidaemia in patients with coronary heart disease: results from the ESC-EORP EUROASPIRE V survey in 27 countries. Atherosclerosis. 2019;285:135-146. doi:10.1016/j.atherosclerosis.2019.03.014

28. Abifadel M, Varret M, Rabès JP, et al. Mutations in PCSK9 cause autosomal dominant hypercholesterolemia. Nat Genet. 2003;34 (2):154-156. doi:10.1038/ng1161.)

29. Stoekenbroek RM, Kallend D, Wijngaard PL, et al. Inclisiran for the treatment of cardiovascular disease: the ORION clinical development program. Future Cardiol. 2018;14(6):433-442. doi:10.2217/fca2018-0067)

30. Ray KK, Wright RS, Kallend D, et al. ORION-10 and ORION-11 Investigators. Two phase 3 trials of inclisiran in patients with elevated LDL Cholesterol. $N$ Engl $J$ Med. 2020;382(16):1507-1519. doi:10.1056/NEJMoa1912387

31. Marrs JC, Anderson SL. Bempedoic acid for the treatment of dyslipidemia. Drugs Context. 2020;9:1-9. doi:10.7573/dic.2020-6-5

32. Lemus HN, Mendivil CO. Adenosine triphosphate citrate lyase: emerging target in the treatment of dyslipidemia. J Clin Lipidol. 2015;9(3):384-389. doi:10.1016/j.jacl.2015.01.002

33. Kosmas CE, Sourlas A, Silverio D, et al. Novel lipid-modifying therapies addressing unmet needs in cardiovascular disease. World $J$ Cardiol. 2019;11(11):256-265. doi:10.4330/wjc.v11.i11.256

34. Esperion therapeutics. A multiple ascending dose study of ETC-1002 in healthy subjects. Available from: https://clinicaltrials.gov/ct2/ show/NCT01485146.

35. Thompson PD, MacDougall DE, Newton RS, et al. Treatment with ETC-1002 alone and in combination with ezetimibe lowers LDL cholesterol in hypercholesterolemic patients with or without statin intolerance. J Clin Lipidol. 2016;10(3):556-567. doi:10.1016/j. jacl.2015.12.025

36. Laufs U, Banach M, Mancini GBJ, et al. Efficacy and safety of bempedoic acid in patients with hypercholesterolemia and statin intolerance. J Am Heart Assoc. 2019;8(7):e011662. doi:10.1161/ JAHA.118.011662

37. Ballantyne CM, McKenney JM, MacDougal DE, et al. Effect of ETC-1002 on serum low-density lipoprotein cholesterol in hypercholesterolemic patients receiving statin therapy. Am $J$ Cardiol. 2016;117(12):1928-1933. doi:10.1016/j.amjcard.2016.03.043

38. Ray KK, Bays HE, Catapano AL, et al. Clear harmony trial. safety and efficacy of bempedoic acid to reduce LDL cholesterol. $N$ Engl $J$ Med. 2019;380(11):1022-1032. doi:10.1056/NEJMoa1803917

39. Ballantyne CM, Laufs U, Ray KK, et al. Bempedoic acid plus ezetimibe fixed-dose combination in patients with hypercholesterolemia and high CVD risk treated with maximally tolerated statin therapy. Eur J Prev Cardiol. 2020;27(6):593-603. doi:10.1177/2047487319864671

40. Reiner Ž. Hypertriglyceridaemia and risk of coronary artery disease. Nat Rev Cardiol. 2017;14(7):401-411. doi:10.1038/nrcardio.2017.31.

41. Reiner Ž. Triglyceride-rich lipoproteins and novel targets for anti-atherosclerotic therapy. Korean Circ J. 2018;48(12):1097-1119. doi:10.4070/kcj.2018.0343

42. Klempfner R, Erez A, Sagit BZ, et al. Elevated triglyceride level is independently associated with increased all-cause mortality in patients with established coronary heart disease: twenty-two-year follow-up of the bezafibrate infarction prevention study and registry. Circ Cardiovasc Qual Outcomes. 2016;9(2):100-108. doi:10.1161/CIRCOUTCOMES.115.002104
43. Bhatt DL, Steg PG, Miller M, et al.; REDUCE-IT Investigators. Cardiovascular risk reduction with icosapent ethyl for hypertriglyceridemia. $N$ Engl $J$ Med. 2019;380(1):11-22. doi:10.1056/NEJMoa1812792.

44. Pećin I, Nedić M, Reiner Ž. Drugs of the future. 2016;41:1-6.

45. Moulin P, Dufour R, Averna M, et al. Identification and diagnosis of patients with familial chylomicronaemia syndrome (FCS): expert panel recommendations and proposal of an "FCS score". Atherosclerosis. 2018;275:265-272. doi:10.1016/j.atheroscl erosis.2018.06.814

46. Witztum JL, Gaudet D, Freedman SD, et al. Volanesorsen and triglyceride levels in familial chylomicronemia syndrome. $N$ Engl J Med. 2019;381(6):531-542. doi:10.1056/NEJMoa1715944

47. Khetarpal SA, Wang M, Khera AV. Volanesorsen, familial chylomicronemia syndrome, and thrombocytopenia. $N$ Engl J Med. 2019;381 (26):2582-2584. doi:10.1056/NEJMc1912350

48. Xu YX, Redon V, Yu H, et al. Role of angiopoietin-like 3 (ANGPTL3) in regulating plasma level of low-density lipoprotein cholesterol. Atherosclerosis. 2018;268:196-206. doi:10.1016/j. atherosclerosis.2017.08.031;

49. Conklin D, Gilbertson D, Taft DW, et al. Identification of a mammalian angiopoietin-related protein expressed specifically in liver. Genomics. 1999;62(3):477-482. doi:10.1006/geno.1999.6041

50. Stitziel NO, Khera AV, Wang X, et al.; PROMIS and Myocardial Infarction Genetics Consortium Investigators. ANGPTL3 deficiency and protection against coronary artery disease. $\mathrm{J} \mathrm{Am} \mathrm{Coll} \mathrm{Cardiol.}$ 2017;69(16):2054-2063. doi:10.1016/j.jacc.2017.02.030.

51. Shimamura M, Matsuda M, Kobayashi S, et al. Angiopoietin-like protein 3, a hepatic secretory factor, activates lipolysis in adipocytes. Biochem Biophys Res Commun. 2003;301(2):604-609. doi:10.1016/ s0006-291x(02)03058-9

52. Wang Y, Gusarova V, Banfi S, et al. Inactivation of ANGPTL3 reduces hepatic VLDL-triglyceride secretion. J Lipid Res. 2015;56 (7):1296-1307. doi:10.1194/jlr.M054882

53. Gaudet D, Gipe DA, Pordy R, et al. ANGPTL3 inhibition in homozygous familial hypercholesterolemia. $N$ Engl J Med. 2017;377 (3):296-297. doi:10.1056/NEJMc1705994

54. Ida S, Kaneko R, Murata K. Efficacy and safety of pemafibrate administration in patients with dyslipidemia: a systematic review and meta-analysis. Cardiovasc Diabetol. 2019;18(1):38. doi:10.1186/s12933-019-0845-x

55. Fruchart JC, Santos RD, Aguilar-Salinas C, et al. The selective peroxisome proliferator-activated receptor alpha modulator (SPPARM $\alpha$ ) paradigm: conceptual framework and therapeutic potential: A consensus statement from the International Atherosclerosis Society (IAS) and the Residual Risk Reduction Initiative (R3i) Foundation. Cardiovasc Diabetol. 2019;18(1):71. doi:10.1186/ s12933-019-0864-7

56. Arai H, Yamashita S, Yokote K, et al. K-877 study group. efficacy and safety of pemafibrate versus fenofibrate in patients with high triglyceride and low hdl cholesterol levels: a multicenter, placebo-controlled, double-blind, randomized trial. $J$ Atheroscler Thromb. 2018;25(6):521-538. doi:10.5551/jat.44412

57. Staels B, Dallongeville J, Auwerx J, et al. Mechanism of action of fibrates on lipid and lipoprotein metabolism. Circulation. 1998;98 (19):2088-2093. doi:10.1161/01.cir.98.19.2088

58. Pradhan AD, Paynter NP, Everett BM, et al. Rationale and design of the pemafibrate to reduce cardiovascular outcomes by reducing triglycerides in patients with diabetes (PROMINENT) study. Am Heart J. 2018;206:80-93. doi:10.1016/j.ahj.2018.09.011

59. Nordestgaard BG, Chapman MJ, Ray K, et al. European atherosclerosis society consensus panel. Lipoprotein(a) as a cardiovascular risk factor: current status. Eur Heart J. 2010;31(23):2844-2853. doi:10.1093/eurheartj/ehq386 
60. Assessing the impact of lipoprotein (a) lowering with TQJ230 on major cardiovascular events in patients with CVD - full text viewClinicalTrials.gov [Internet]. Available from: https://clinicaltrials. gov/ct2/show/NCT04023552. Accessed November 27, 2020

61. Stiekema LCA, Prange KHM, Hoogeveen RM, et al. Potent lipoprotein(a) lowering following apolipoprotein(a) antisense treatment reduces the pro-inflammatory activation of circulating monocytes in patients with elevated lipoprotein(a). Eur Heart J. 2020;41 (24):2262-2271. doi:10.1093/eurheartj/ehaa171

62. Wei T, Cheng Q, Min YL, et al. Systemic nanoparticle delivery of CRISPR-Cas9 ribonucleoproteins for effective tissue specific genome editing. Nat Commun. 2020;11(1):3232. doi:10.1038/s41467-02017029-3
63. Sun W, Wang J, Hu Q, et al. CRISPR-Cas $12 \mathrm{a}$ delivery by DNA-mediated bioresponsive editing for cholesterol regulation. Sci Adv. 2020;6(21):eaba2983. doi:10.1126/sciadv.aba2983

64. Reiner $\check{Z}$. Can Lp(a) lowering against background statin therapy really reduce cardiovascular risk? Curr Atheroscler Rep. 2019;21 (4):14. doi:10.1007/s11883-019-0773-y

65. Chadwick AC, Evitt NH, Lv W, et al. Reduced blood lipid levels with in vivo CRISPR-Cas9 base editing of ANGPTL3. Circulation. 2018;137:975-977. doi:10.1161/CIRCULATIONAHA.117.031335

\section{Publish your work in this journal}

The Journal of Experimental Pharmacology is an international, peerreviewed, open access journal publishing original research, reports, reviews and commentaries on all areas of laboratory and experimental pharmacology. The manuscript management system is completely online and includes a very quick and fair peer-review system. Visit http://www.dovepress.com/testimonials.php to read real quotes from published authors. 